\title{
MISTAKES IN DEALING WITH AORTIC DISSECTION. LESSONS FROM THREE WARNING CASES.
}

\author{
Dan Marek $^{\mathrm{a} *}$, Petr Nemec ${ }^{\mathrm{b}}$, Miroslav Herman ${ }^{\mathrm{c}}$, Marek Gwozdziewicz ${ }^{\mathrm{b}}$, Martin Troubil ${ }^{\mathrm{b}}$, \\ Jan Lukl ${ }^{\mathrm{a}}$
}

\author{
a Department of Internal Medicine, University Hospital Olomouc, Czech Republic \\ b Department of Cardiosurgery, University Hospital Olomouc \\ c Department of Radiology, University Hospital Olomouc \\ e-mail:audiodan@centrum.cz
}

Received: September 26, 2008; Accepted: October 23, 2008

Key words: Aortic dissection/Complications/Diagnosis/Treatment/Mistakes

Background: Aortic dissection is a dangerous condition with a high mortality in the acute stage. Aortic dissection requires early diagnosis and treatment.

Methods and Results: This short review discusses and focuses on known complications of aortic dissection and its natural mortality applying data from already published reports and from cohorts and registers, especially IRAD. Survival data of patients with type A and type B of dissection are presented and treatment options are proposed. The review presents three interesting cases from our database pointing out mistakes made in the diagnostic process and in dealing with the patient even after establishing the correct diagnosis. In one case, a patient with chest pain + "immeasurable" BP was suspected to suffer from an acute myocardial infarction and cardiogenic shock instead of AoD + aortic branch obstruction. In another patient with chest pain + V1V2 ST elevation, again the acute coronary syndrome was suspected. In fact, AoD with a perforation to cardiac chambers through the interventricular septum was the explanation. In the third case, the correct diagnosis of AoD was established. This patient was at a significant risk of aortic rupture because of his uncontrolled blood pressure. Instead of sedation administration and effective BP lowering, the patient was stressed even more by detailed information about this life threatening disease. This led to an aortic rupture with cardiac tamponade. Other mistakes made when dealing with all these presented cases are also discussed.

Conclusion: The high mortality in patients suffering from aortic dissection is often potentiated by misdiagnosing and mishandling of these patients in clinical scenario.

\section{INTRODUCTION}

Aortic dissection (AoD) is a life threatening disease. Its natural mortality is high ( $1 \%$ in 1 hour during the first 48 hours, if untreated) (ref. ${ }^{1}$ ). Most patients with AoD have hypertension $(80 \%)$ in their histories ${ }^{2,3}$ or there is a defective aortic tissue found at autopsy (Marfan syndrome, Ehlers Danlos syndrome). Physical effort or emotional stress elevating pulse pressure is a common trigger of acute AoD. A sudden sharp pain is a leading symptom of AoD but it is not necessarily present in all cases. Other signs of AoD come from branch obstruction (myocardial infarction, stroke, bowel ischemia, paraplegia etc.), acute heart failure (due to aortic regurgitation or arrhythmias) or rupture and bleeding (hemothorax, hemopericardium, retro/intraperitoneal hematoma). Disseminated intravascular coagulation is common. Multiorgan failure (due to either arterial obstruction or disseminated intravascular coagulation) could cause death in the subacute period ${ }^{3}$.

Management of patients with AoD requires early diagnosis and treatment. According to the guidelines ${ }^{4}$, pain relief by morphine and blood pressure reduction are the treatment goals in the early stage of management. A target systolic blood pressure of about 100-120 mms of mercury should be achieved and maintained by medication (betablockers \pm vasodilators). According to the Stanford classification, in type A AoD the dissection involves the ascending aorta. In type $\mathrm{B}$, the proximal aorta is not involved $^{5}$. It is very important to distinguish between type A and $\mathrm{B}$ AoD, as in type A an urgent operation is indicated. In type $\mathrm{B}$, a medical approach is advocated.

Even if the correct diagnosis is made quickly and the treatment delivered properly, the number of complications potentially leading to death is high. Hagan ${ }^{6}$ published an extensive observation of 464 patients with AoD. The data came from the contemporary, multi-center International Registry of Acute Aortic Dissection (IRAD), collecting patients from 18 referral centers worldwide. Among these, $62.3 \%$ had type A dissection. Overall in-hospital mortality was $27.4 \%$. The mortality of patients with type A dissection managed surgically was $26 \%$; among those not receiving surgery (typically because of advanced age and co-morbidity), mortality was $58 \%$. Mortality of patients with type B dissection treated medically was $10.7 \%$. Surgery was performed in $20 \%$ of patients with type B dissection; mortality in this group was $31.4 \%$. Patients with coronary involvement, other vascular complications or presenting with neurological symptoms demonstrate the highest mortality. Such an "unstable" group of patients (presence of cardiac tamponade, shock, congestive heart 
failure, cerebrovascular accident, stroke, coma, myocardial ischemia or infarction, electrocardiograms with new $Q$ waves or ST elevation, acute renal failure or mesenteric ischemia-infarction at the time of the operation) had mortality $31.4 \%$ in IRAD. The "stable" group had mortality $16.7 \%$. Compared to the "unstable" group, the difference was significant $(\mathrm{p}<0.001)\left(\right.$ ref. $\left.^{7}\right)$. Once the patient survives an acute stage, the prognosis improves dramatically. In the study published by Rizzoli, the maximum followup in 119 patients operated on for acute type A AoD was 11.7 years (median 5.6 years). The survival rate in this cohort was $47.3 \%+/-5 \%$. The death risk decreased rapidly to a constant rate of 0.0027 events/month after three months ${ }^{8}$. Tsai used data from IRAD to elucidate the long-term survival of patients with type A AoD after their hospital discharge and to identify the predictors of death. They examined 303 consecutive patients. Two hundred and seventy three $(90.1 \%)$ of them were managed surgically and 30 (9.9\%) were managed medically. The Kaplan-Meier survival curves were constructed to depict cumulative survival in patients from the date of hospital discharge. Survival of patients treated with surgery was $96.1 \%+/-2.4 \%$ and $90.5 \%+/-3.9 \%$ at 1 and 3 years, respectively, versus $88.6 \%+/-12.2 \%$ and $68.7 \%+/-19.8 \%$ without surgery (mean follow-up was 2.8 years) ( ref. $^{9}$ ).

Therefore, avoidance of misdiagnosis and/or improper treatment in the acute stage is essential in dealing with AoD patients to retain the mortality as low as possible.

Unfortunately, in the clinical scenario, the high mortality is often due to misdiagnosing AoD. AoD is a relatively rare cause of chest pain which makes the situation even worse - not many doctors managing such a patient consider the diagnosis of AoD on first contact. Meszáros revealed that the dissection of the aorta was diagnosed in vivo only in 22 out of the 75 patients who died in hospital (29\%). Twenty other patients (21\% of 95) died before $\operatorname{admission}^{10}$.

We present three illustrative cases, where we demonstrate some mistakes in the diagnostic process and treatment of AoD.

\section{Case report 1}

A 68-year old female collapsed in a pharmacy within the area of a big tertiary center, but she remained conscious and reported a sharp chest pain. When an emergency team came, the doctor was not able to measure blood pressure or palpate any peripheral arterial pulsation on her right arm. Since the patient had hypertension and myocardial infarction in her history, the doctor assumed that acute myocardial infarction with incipient cardiogenic shock was the diagnosis. Benzodiazepines alone were administered and the patient was transferred directly to CCU for further management. On admission to the CCU, there was a clear difference in arterial pulsations between the right and the left arms. A carotid murmur and systolic/diastolic murmur upon the aortic valve region were present. These findings led to a suspicion of AoD, which was confirmed promptly by bedside transoesophageal echocardiography (type A AoD, Fig. 1).

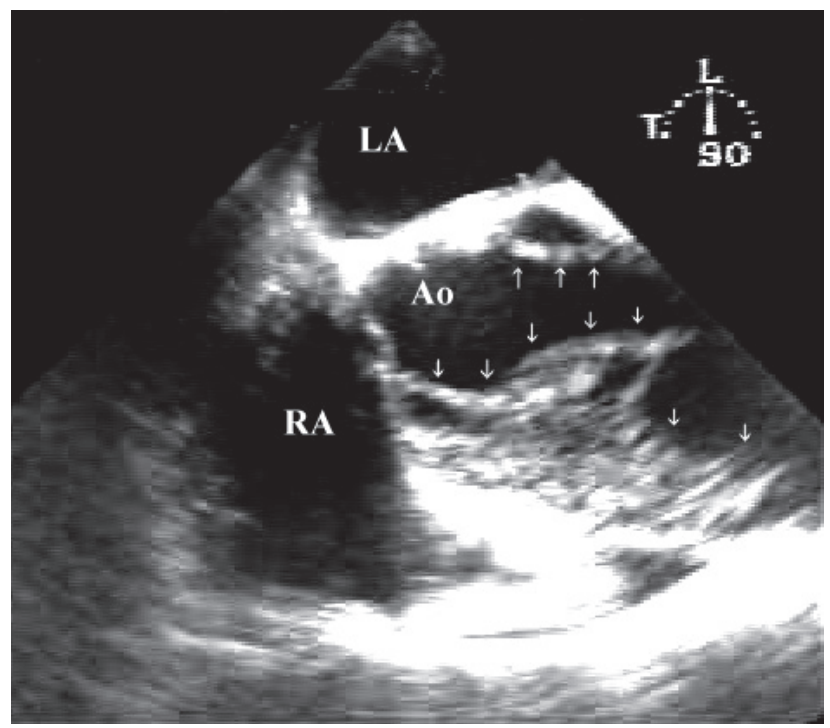

Fig. 1. Case 1. TOE image of the dissected ascending aorta (Ao). Floating intimal flaps are marked by arrows. (LA - left atrium, RA - right atrium).

The patient was successfully operated on but she died from multiorgan failure due to disseminated intravascular coagulation four days later.

\section{Discussion}

1. One could hardly expect normal consciousness if the "immeasurable blood pressure" is genuinely due to severe hypotension. This "mismatch" should lead to further careful examination of the circulation system.

2. A difference in pulse quality upon great arteries is a pathognomonic and useful sign of AoD, especially in prehospital care settings. The systolic and diastolic murmurs are supporting symptoms.

3. Incomplete physical examination without checking the circulatory system properly was the essential mistake, leading to a false hypothesis of acute MI. Fortunately, no antiaggregation, anticoagulation or thrombolytic treatment was given in this particular patient.

4. Atherosclerosis is a risk factor not only in ischemic heart disease but also in AoD. Therefore, its presence does not help much in the differentiation between these two dangerous entities.

\section{Case report 2}

A 70-year old man with hypertension and pneumoconiosis in his history had a syncopal episode when working physically at his garden. This was witnessed by his wife. In a couple of minutes, he became conscious but suffered from an intensive sharp chest pain, and he had lower limb paraplegia and left upper limb paresis. No rescue team was called and the family transferred the patient to the tertiary center 3 hours later - after his symptoms had disappeared partly. On admission to the emergency room, the patient was conscious, hypotensive (systolic BP $80 \mathrm{~mm}$ of mercury) and his peripheral arterial pulsations were symmetrical. A systolic murmur $2 / 6$ in the $3^{\text {rd }}$ right parasternal 


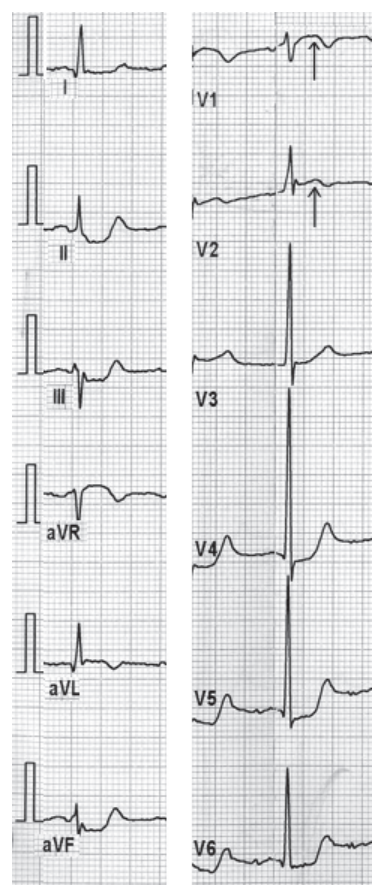

Fig. 2. Case 2. ECG shows a mild elevation of ST segment in V1 and V2 (arrows) with contralateral ST depressions in II, III and VF, indicating presence of myocardial necrosis.

intercostal space was detected. No paresis was present. There were signs of septal myocardial infarction on ECG (ST segment elevations) in V1 and V2 (Fig. 2). Elevated troponin $\mathrm{T}$ was detected in the serum. The diagnosis of myocardial infarction complicated by brain embolization from the infarction site was assumed and the patient was admitted to a CCU (the suggested plan was to proceed with direct coronary angioplasty). At the $\mathrm{CCU}$, aortic dissection was suspected (based on the history) and a CT scan confirmed type A AoD (Fig. 3). The descending aorta was also found dissected almost to the bifurcation. Both false and true lumina were patent. Perioperative transoesophageal echocardiography (TOE) discovered a very rare complication: a dissected ventricular septum with a shunt to the right ventricle (Fig. 4), which progressed quickly and became hemodynamically significant. The latter condition led to a cardiogenic shock, and despite the fact that the patient was operated on, he died on the second postoperative day due to multiorgan failure. At autopsy, perforation of the interventricular septum surrounded by a hemorhagic infarction was confirmed. No obstructive intracoronary lesion was found.

\section{Discussion}

1. The fact that the acute chest pain developed simultaneously with neurological signs made the diagnosis of brain embolization from the infarction site rather doubtful. If an acute MI with left ventricular hypokinesia and subendocardial necrosis develops, it usually takes some time (at least hours) before any thrombus on the endocardial inflammation site can form.

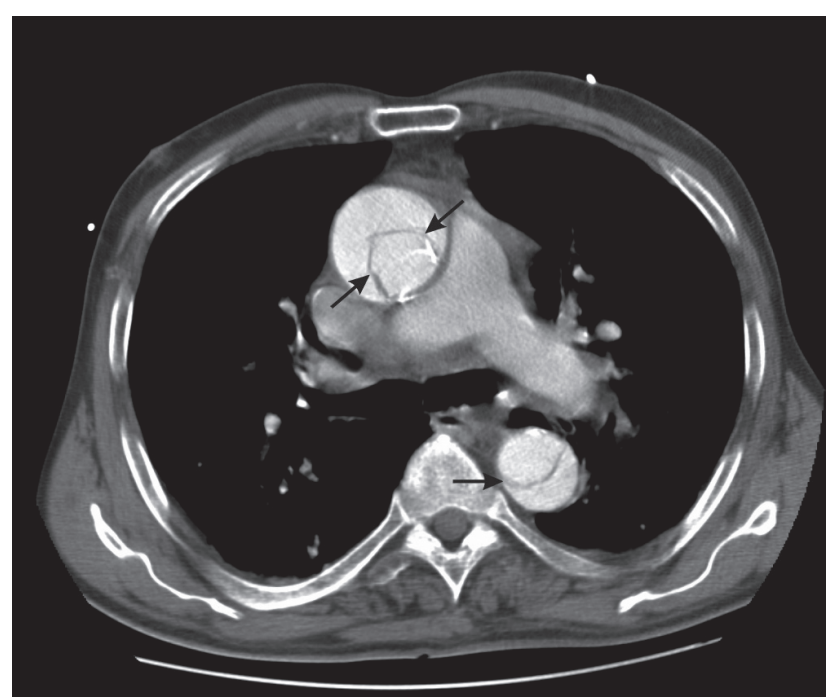

Fig. 3. Case 2. CT scan. Both ascending (double arrow) and descending aorta (single arrow) are dissected.

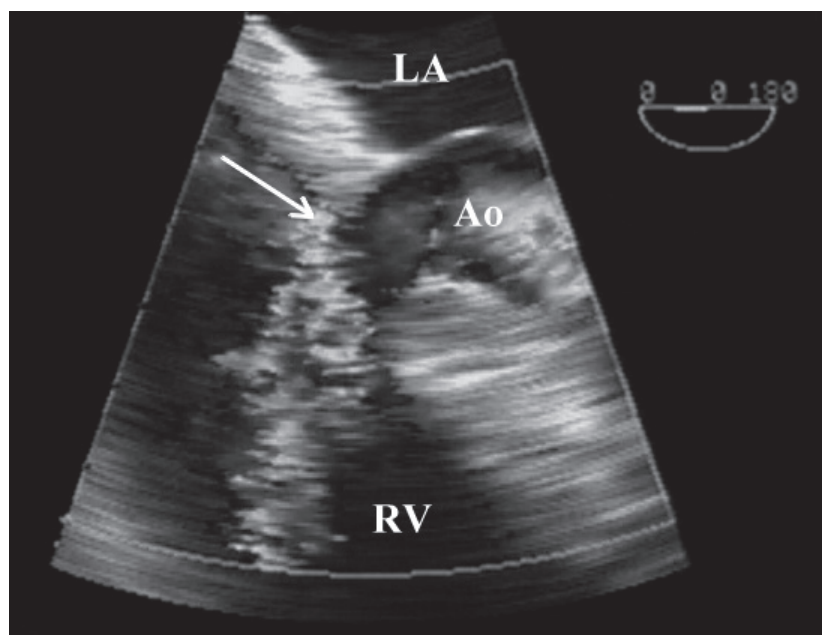

Fig. 4. Case 2. TOE image of the shunt from the aorta to the right ventricle (collor Doppler flow - arrow) through the interventricular septum $(\mathrm{RV}$ - right ventricle, Ao - aorta, LA - left atrium).

2. Obstruction of the left coronary artery orifice by an intimal flap usually leads to a large anterolateral infarction, and, in such a case, extensive ECG changes can be expected. In this patient, there were just small ST elevations in two ventral leads (V1 and V2) present on admission. Therefore, in the presented case, an intramural septal mechanical disruption came first and it was followed by an infarction of the muscle tissue around the rupture. This myocardial necrosis was expressed as the ST elevation. The positivity of troponin is not surprising in myocyte damage.

3. Again, fortunately, no anticoagulation and antiaggregation therapy had been administered before the correct diagnosis was made. Otherwise, there could have been a debate about making the prognosis even worse. 
4. Both false and true lumina were patent in the thoracic aorta. This explains the fact that symmetrical peripheral arterial pulsations were palpable in this patient. Although missing peripheral pulsation is a pathognomonic sign of AoD, it is not necessarily present in all patients (in fact, it is been reported only in $15 \%$ of the cases registered in IRAD) (ref. ${ }^{6}$ ).

5. Rupture of the aortic root with a shunt directly into cardiac chambers is a rare complication, usually leading to acute heart failure. Some cases have been published in the literature, with the right atrium being most frequently targeted.

6. One could speculate that the long delay caused by reluctance of the patient's relatives to call an emergency team made the prognosis much worse. In any event, the vascular damage in the aorta was huge, and, according to the CT scan, abdominal organs were targeted as well. Even without the septal rupture and the right to left shunt, the survival chance was low.

\section{Case report 3}

A 53-year old male was admitted to a district hospital because of severe back pain after falling down. On admission, he was hypotensive. At a general surgical unit, he was treated as having a traumatic back pain. After a couple of inconclusive tests and examinations, other causes of chest pain came under considerations. A physician suspected an AoD. To confirm the diagnosis by a transoesophageal echocardiography, the patient was transferred to a tertiary center after three days. On arrival to the echocardiographic laboratory, the patient was very scared and agitated, with a blood pressure of 160/90. Even in a transthoracic view, it was clear that AoD was the correct diagnosis: fluttering intimal flaps were visible within the aortic root and the aortic arch (Fig. 5). The patient was informed about this diagnosis and about the need of an urgent operation. An instant TOE was also suggested to elucidate the character and extension of AoD. At that moment, the patient reported a "sudden sickness" and lost consciousness in seconds. Immediate repeated transthoracic view revealed a huge hemopericardium with tamponade. Any resuscitating effort made (including urgent pericardiocentesis) to save the patient's life was ineffective. AoD with perforation was confirmed at autopsy. Four hundred milliliters of blood were present in the pericardial cavity according to the report.

\section{Discussion}

1. Again, the more one thinks about an AoD, the sooner the diagnosis is made.

2. In this case, eventually the correct diagnosis of AoD was suspected. The patient survived the first 48 hours which made his prognosis much better. Despite these facts, a fatal mistake was probably made in handling the patient with AoD confirmed by transthoracic echocardiogram.

This patient was at significant risk of aortic rupture because of his uncontrolled blood pressure. When AoD was seen on transthoracic echo in this agitated patient, a

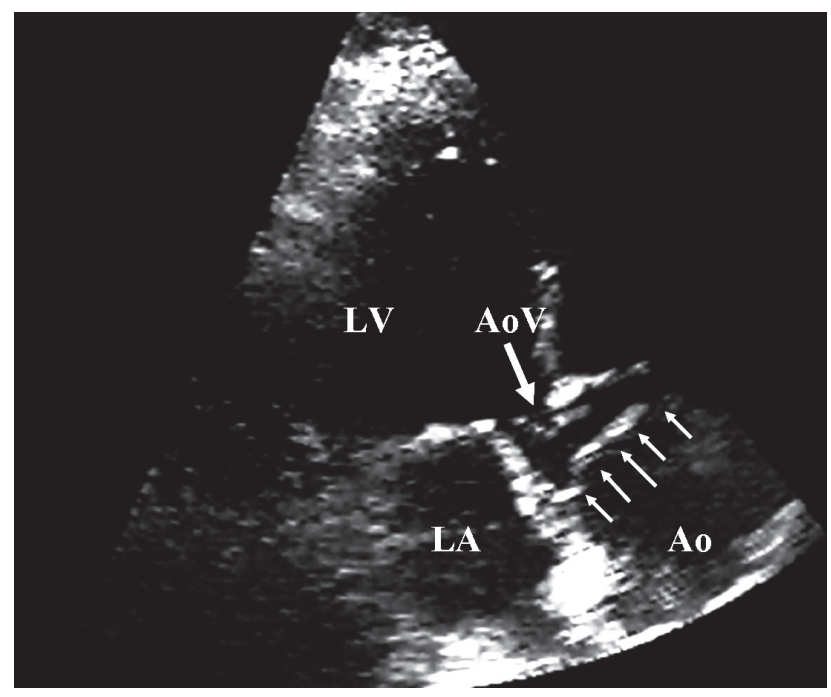

Fig. 5. Case 3. Apical image shows a dissected intimal flap in the aortic root (multiple arrows). Aortic valve (AoV, single arrow) is moderately calcified. (LV - left ventricle, Ao - aorta, LA - left atrium).

different approach should have been adopted in this situation: first - pain relief and sedation of the patient, second - effective decrease in patient's systolic blood pressure, third - finally, completing the examination by TOE or any other relevant diagnostic modality. Patient's informed consent would not be required in this emergency. Instead of the actions proposed above, the patient was stressed even more by detailed information about the life threatening disease and forthcoming operation.

In summary, according to the European Society of Cardiology guidelines ${ }^{4}$, the initial management of patients with suspected aortic dissection should consist of further steps:

- Detailed medical history and complete physical examination, transfer to intensive care unit

- Intravenous line, blood sample (CK, TnT(I), myoglobin, WBC, D-dimer, haematocrit, $\mathrm{LDH}$ )

- ECG: documentation of ischaemia

- Heart rate and blood pressure monitoring

- Pain relief (morphine sulphate)

- Reduction of systolic blood pressure using beta-blockers (i.v. propranolol, metoprolol, esmolol or labetalol). In patients with severe hypertension additional vasodilator (i.v. sodium nitroprusside to titrate BP to $100-120 \mathrm{mmHg}$ ). In patients with obstructive pulmonary disease, blood pressure lowering with calcium channel blockers

- Imaging modalities to confirm or exclude AoD (especially in patiens with signs of ischaemia on ECG) before thrombolysis, anticoagulation or antiaggregation are given. Every patient with suspected aortic dissection should undergo diagnostic imaging (CT, NMR, TOE) to rule out the disease. The benefits of this strategy outweigh the risks of being harmful to patients ultimately found to have another condition. 
Further therapeutic solutions depend on the results of the diagnostic process. As stated above, urgent operation is indicated in type A AoD. Patients with type B AoD should be managed conservatively.

\section{REFERENCES}

1. Hirst AE, Johns VJ Jr, Kime SW Jr. Dissecting aneurysm of the aorta: review of 505 cases. Medicine 1958; 37:217-279.

2. Kaplan NM. Clinical hypertension 7th ed. Baltimore; 1998. p. 114.

3. Isselbacher EM, Eagle KA, Desanctis RW. Diseases of the Aorta In: Braunwald E (ed). Heart Disease, Saunders Company, USA; 1997. p. 1558-64.

4. Erbel R, Alfonso F, Boileru C, Dirsch O, Eber B, Haverich A, Rakowski H, Struyven J, Radegran K, Fechtem U, Tailor J, Zollikofer Ch. Diagnosis and management of aortic dissection. Recommendations of the Task Force on Aortic Dissection, European Society of Cardiology. Eur Heart J 2001; 22:1642-681.

5. Daily PO, Trueblood HW, Stinson EB, Wuerflein RD, Shumway NE. Managment of acute aortic dissection. Ann Thorac Surg 1970; $10: 237$.
6. Hagan PG, Nienaber CA, Isselbacher EM, Bruckman D, Karavite DJ, Russman PL, Evangelista A, Fattori R, Suzuki T, Oh JK, Moore AG, Malou JF, Pape LA, Gaca C, Fechtem U, Lenferink S, Deutsch HJ, Diedrichs H, Nobles JMY, Llovet A, Gilon D, Das SK, Armstrong WF, Deeb GM, Eagle KA. The International Registry of Acute Aortic Dissection (IRAD) - New insights into an old disease. JAMA 2000; 283:897-903.

7. Trimarchi S, Nienaber CA, Rampoldi V, Myrmel T, Suzuki T, Mehta RH, Bossone E, Cooper JV, Smith DE, Menicanti L, Frigiola A, Oh JK, Deeb MG, Isselbacher EM, Eagle KA. Contemporary results of surgery in acute type A aortic dissection: The International Registry of Acute Aortic Dissection experience. J Thorac Cardiovasc Surg 2005; 129:112-22.

8. Rizzoli G, Mazzucco A, Fracasso A, Giambuzzi M, Rubino M, Gallucci V. Early and late survival of repaired type A aortic dissection. Eur J Cardiothorac Surg 1990; 4:575-83.

9. Tsai TT, Evangelista A, Nenaber CA, Trimarchi S, Fechtem U, Fattori R, Myrmel T, Pape L, Cooper JV, Smith DE, Fang JM, Isselbacher E, Eagle KA. Long-term survival in patients presenting with type A acute aortic dissection - Insights from the International Registry of Acute Aortic Dissection (IRAD). Circulation 2006; 114:I350-I356.

10. Meszaros I, Morocz J, Szlavi J, Schmidt J, Nagy L, Kato C, Tornoci L. Neurologic complications of aortic dissection. Ideggyogy.Sz 2002; 55:148-55 (abstract). 
